\title{
Qualitative research essentials for medical education
}

\author{
Sayra M Cristancho $^{1,2}$, PhD, Mark Goldszmidt ${ }^{2,3}, \mathrm{MD}, \mathrm{PhD}$, Lorelei $\underline{\text { Lingard }}^{2,3}$, PhD, Christopher Watling ${ }^{2,4}, \mathrm{MD}, \mathrm{PhD}$
}

This paper offers a selective overview of the increasingly popular paradigm of qualitative research. We consider the nature of qualitative research questions, describe common methodologies, discuss data collection and analysis methods, highlight recent innovations and outline principles of rigour. Examples are provided from our own and other authors' published qualitative medical education research. Our aim is to provide both an introduction to some qualitative essentials for readers who are new to this research paradigm and a resource for more experienced readers, such as those who are currently engaged in a qualitative research project and would like a better sense of where their work sits within the broader paradigm.

Keywords: medical education, methodology, qualitative research

\section{INTRODUCTION}

Are you a medical education researcher engaged in qualitative research and wondering if you are on the right track? Are you contemplating a qualitative research project and not sure how to get started? Are you reading qualitative manuscripts and making guesses about their quality? This paper offers a selective overview of the increasingly popular domain of qualitative research. We consider the nature of qualitative research questions, describe common methodologies, discuss data collection and analysis methods, highlight recent innovations, and outline principles of rigour. The aim of this paper is to educate newcomers through introductory explanations while stimulating more experienced researchers through attention to current innovations and emerging debates.

\section{WHAT IS QUALITATIVE RESEARCH?}

Qualitative research is naturalistic; the natural setting - not the laboratory - is the source of data. Researchers go where the action is; to collect data, they may talk with individuals or groups, observe their behaviour and their setting, or examine their artefacts. ${ }^{(1)}$ As defined by leading qualitative researchers Denzin and Lincoln, qualitative research studies social and human phenomena in their natural settings, attempting to make sense of or interpret these phenomena in terms of the meanings participants bring to them. ${ }^{(2)}$

Because qualitative research situates itself firmly in the world it studies, it cannot aim for generalisability. Its aim is to understand, rather than erase, the influence of context, culture and perspective. Good qualitative research produces descriptions, theory or conceptual understanding that may be usefully transferred to other contexts, but users of qualitative research must always carefully consider how the principles unearthed might unfold in their own distinct settings.

\section{Box 1. Qualitative research questions:}

- Explore social process and human experience

- Ask about what, why and how

- Seek to explore and explain

\section{WHAT QUESTIONS ARE APPROPRIATE FOR QUALITATIVE RESEARCH?}

Meaningful education research begins with compelling questions. Research methods translate curiosity into action, facilitating exploration of those questions. Methods must be chosen wisely; some questions lend themselves to certain methodological approaches and not to others.

In recent years, qualitative research methods have become increasingly prominent in medical education. The reason is simple: some of the most pressing questions in the field require qualitative approaches for meaningful answers to be found.

Qualitative research examines how things unfold in real world settings. While quantitative research approaches that dominate the basic and clinical sciences focus on testing hypotheses, qualitative research explores processes, phenomena and settings (Box 1). For example, the question "Does the introduction of a mandatory rural clerkship increase the rate of graduates choosing to practise in rural areas?" demands a quantitative approach. The question embeds a hypothesis - that a mandatory rural clerkship will increase the rate of graduates choosing to practise in rural areas - and so the research method must test whether or not that hypothesis is true. But the question "How do graduating doctors make choices about their practice location?" demands a qualitative approach. The question does not embed a hypothesis; rather, it explores a process of decision-making.

Many issues in medical education could be examined from either a quantitative or qualitative approach; one approach is not inherently superior. The questions that drive the research as well

${ }^{1}$ Department of Surgery and Faculty of Education, ${ }^{2}$ Centre for Education Research and Innovation, ${ }^{3}$ Department of Medicine, ${ }^{4}$ Postgraduate Medical Education, Schulich School of Medicine and Dentistry, Western University, Canada

Correspondence: Dr Lorelei Lingard, Director, Centre for Education Research and Innovation, Schulich School of Medicine and Dentistry, Western University, Room H112, Health Sciences Addition, London, Ontario, Canada N6A 5C1. Lorelei.lingard@schulich.uwo.ca 
as the products that derive from it are, however, fundamentally different. Consider two approaches to studying the issue of online learning. A quantitative researcher might ask, "What is the effect of an online learning module on medical students' end-of-semester OSCE [objective structured clinical examination] scores?", while a qualitative researcher might ask "How do medical students make choices about using online learning resources?" Although the underlying issue is the same - the phenomenon of online learning in medical school - the studies launched by these questions and the products of those studies will look very different.

\section{WHAT ARE QUALITATIVE METHODOLOGIES AND WHY ARE THEY IMPORTANT?}

Executing rigorous qualitative research requires an understanding of methodology - the principles and procedures that define how the research is approached. Far from being monolithic, the world of qualitative research encompasses a range of methodologies, each with distinctive approaches to inquiry and characteristic products. Methodologies are informed by the researcher's epistemology - that is, their theory of knowledge. Epistemology shapes how researchers approach the researcher's role, the participant-researcher relationship, forms of data, analytical procedures, measures of research quality, and representation of results in analysis and writing. ${ }^{(3)}$

In medical education, published qualitative work includes methodologies such as grounded theory, phenomenology, ethnography, case study, discourse analysis, participatory action research and narrative inquiry, although the list is growing as the field embraces researchers with diverse disciplinary backgrounds. This paper neither seeks to exhaustively catalogue all qualitative methodologies nor comprehensively describe any of them. Rather, we present a subset, with the aim of familiarising readers with its fundamental goals. In this article, we briefly introduce four common methodologies used in medical education research (Box 2). Using one topic, professionalism, we illustrate how each methodology might be applied and how its particular features would yield different insights into that topic.

\section{Grounded theory}

Arguably the most frequently used methodology in medical education research today, grounded theory seeks to understand social processes. Core features of grounded theory include iteration, in which data collection and analysis take place concurrently with each informing the other, and a reliance on theoretical sampling to explore patterns as they emerge. ${ }^{(4)}$ While many different schools of grounded theory exist, they share the aim of generating theory that is grounded in empirical data. ${ }^{(5)}$ Theory, in this type of research, can be thought of as a conceptual understanding of the process under study, ideally affording a useful explanatory power. For example, if one were interested in the development of professionalism among senior medical students during clerkship, one might design a grounded theory study around the following question: "What aspects of clerkship support or challenge professional behaviour among

\section{Box 2. Common qualitative methodologies in medical education:}

1. Grounded theory: explores social processes to produce explanatory theory

2. Phenomenology: deeply probes individual experiences of selected phenomenon

3. Ethnography: studies culture to describe the nature and meanings of routines and rituals

4. Case study: analyses one (or more) instances of a problem, to inform understanding

senior medical students?" The resulting product would be a conceptual rendering of how senior medical students navigate thorny professionalism issues, which might in turn be useful to curriculum planners.

\section{Phenomenology}

This methodology begins with a phenomenon of interest and seeks to understand the subjective lived experience of that phenomenon. ${ }^{(6)}$ Core features of phenomenology include a focus on the individual experience (typically pursued through in-depth interviewing and/or examinations of personal narratives), inductive analysis and a particular attention to reflexivity. ${ }^{(7)}$ Phenomenological researchers typically enumerate their own ideas and preconceptions about the phenomenon under study and consider how these perceptions might influence their interpretation of data. ${ }^{(8)}$ A phenomenological study around professionalism in senior medical students, for example, might involve interviewing several students who have experienced a professionalism lapse about that experience. The resulting product might be an enhanced understanding of the emotional, social and professional implications of this phenomenon from the student's perspective, which might in turn inform wellness or resilience strategies.

\section{Ethnography}

Ethnography aims to understand people in their contexts, exploring the influence of culture, social organisation and shared values on how people behave - their routines and rituals. Core features of ethnography include reliance on direct observation as a data source, and the use of sustained immersive engagement in the setting of interest in order to understand social dynamics from within. ${ }^{(9,10)}$ An ethnographic approach to studying how professional attitudes develop in senior medical students might gather data through observations of ward rounds, team meetings and clinical teaching sessions over a period of time. The resulting product - called an ethnography - would describe how professional values are socialised in junior learners in clinical settings, which could assist educators in understanding how the clinical experiences they programme for their learners are influencing their professional development.

\section{Case study}

Case study research seeks an in-depth understanding of an individual case (or series of cases) that is illustrative of a problem 
of interest. Like clinical case studies, the goal is not generalisation but a thorough exploration of one case, in hopes that the fruits of that exploration may prove useful to others facing similar problems. Core features of case study research include: thoughtful bounding or defining of the scope of the case at the outset; collection of data from multiple sources, ranging from interviews with key players to written material in policy documents and websites; and careful attention to both the phenomenon of interest and its particular context. ${ }^{(11)} \mathrm{A}$ specific professionalism challenge involving medical students could provide fodder for a productive case study. For example, if a medical school had to discipline several students for inappropriately sharing personal patient information on social media, a case study might be undertaken. The 'case' would be the incident of social media misuse at a single medical school, and the data gathered might include interviews with students and school officials, examination of relevant policy documents, examination of news media coverage of the event, and so on. The product of this research might trigger similar institutions to carefully consider how they might approach - or prevent - a similar problem.

As these four examples illustrate, methodology is the backbone of qualitative research. Methodology shapes the way the research question is asked, defines the characteristics of an appropriate sample, and governs the way the data collection and analysis procedures are organised. The researcher's role is also distinctive in each methodology; for instance, in constructivist grounded theory, the researcher actively constructs the theory, ${ }^{(12)}$ while in phenomenology, the researcher attempts to manage his or her 'pre-understandings' through either bracketing them off or being reflexive about them. ${ }^{(13)}$ Interested readers may wish to consult the reference list for recently published examples of research using grounded theory, ${ }^{(14)}$ phenomenology, ${ }^{(15)}$ ethnography ${ }^{(16)}$ and case study approaches ${ }^{(17)}$ in order to appreciate how researchers deploy these methodologies to tackle compelling questions in contemporary medical education.

\section{WHAT ARE SOME COMMON METHODS OF QUALITATIVE DATA COLLECTION?}

The most common methods of qualitative data collection are interview - talking to participants about their experiences relevant to the research question, and observation - watching participants while they are having those experiences. Depending on the research questions explored, a research design might combine interviews and observations.

\section{Interview-based methods}

Interviews are typically used for situations where a guided conversation with relevant participants can help provide insight into their lived experiences and how they view and interpret the world around them. Interviews are also particularly useful for exploring past events that cannot be replicated or phenomena where direct observation is impossible or unfeasible.

Participants may be interviewed individually or in groups. Focus group interviews are used when the researcher's topic of interest is best explored through a guided, interactive discussion among the participants themselves. Therefore, when focus groups are used, the sample is conceptualised at the level of the group - three focus groups of five people constitutes a sample of three interactive discussions, not 15 individual participants. Because they centre on the group discussion and dynamic, focus groups are less wellsuited for topics that are sensitive, highly personal or perceived to be culturally inappropriate to discuss publicly. ${ }^{(18)}$

Unlike quantitative interviews, where a set of structured, closed-ended (e.g. yes/no) questions are asked in the same order with the same wording every time, qualitative interviews typically involve a semi-structured design where a list of openended questions serves to guide, but not constrain, the interview. Therefore, at the interviewer's discretion, the questions and their sequence may vary from interview to interview. This judgement is made based on both the interviewer's understanding of the phenomenon under exploration and the emerging dynamic between the interviewer and participant.

The primary goal of a qualitative interview is to get the participants to think carefully about their experience and relate it to the interviewer with rich detail. Getting good data from interviewing relies on using creative strategies to avoid the common trap of getting politically correct answers - often called 'cover stories' - or answers that are superficial rather than deep and reflective. ${ }^{(19)} \mathrm{A}$ common design error occurs when researchers are overly explicit in their questioning, such as asking "What are the top five criteria you use to assess student professionalism?" A better approach involves questions that ask participants to describe what they do in practice, with follow-up probes that extend beyond the specific experience described. For example, starting with "Tell me about a recent experience where you assessed a student's professionalism" allows the participant to relay an experience, to which the interviewer can respond with probes such as "What was tricky about that?" or "How typical is that experience?"

Another common strategy for prompting participants to engage in rich reflection on their experience and perceptions is to use vignettes as discussion prompts. Vignettes are often artificial scenarios presented to participants to read or watch on video, about which they are then asked probing questions. ${ }^{(20)}$ However, vignettes can also be used to recreate an authentic situation for the participant to engage with. ${ }^{(21)}$ For instance, in one interview study, we presented participants with a vignette in the form of the research assistant reading aloud a standard patient admission presentation that the interviewees would typically hear from their students on morning ward rounds. We then asked the participants to interact with the interviewer as though he or she was a student who had presented this case on morning rounds. Recreating this interaction in the context of the interview served as a stepping stone to questions such as "Why did you ask the student ' $x$ '?" and "How would your approach have differed with a different student presenter, e.g. a stronger or weaker one?"

\section{Direct observation}

Observation-based research can involve a wide spectrum of activities, ranging from brief observations of specific tasks 
(e.g. handover, preoperative team briefings) to prolonged field observations such as those seen in ethnography. When used effectively, direct observation can provide the researcher with powerful insight into the routines of a group.

Getting good data from observational research relies on several key components. First, it is essential to define the scope of the project upfront: limited budgets, the massive amount of detail to be attended to, and the ability of any individual or group of observers to attend to these make this essential. Good observational research therefore relies on collaboration between knowledgeable insiders and those with both methodological and theoretical expertise. Sampling demands particular attention; an initial purposive sampling approach is often followed by more targeted, theoretical sampling that is guided by the developing analysis. Observational research also typically involves a mix of data sources, including observational field notes, field interviews and document analysis. Audio and video may be helpful when the studied phenomena is particularly complex or nuances of interaction may be missed without the ability to review data, or when precision of verbal and nonverbal interactions is necessary to answer the research question. ${ }^{(22)}$

Field notes are often the dominant data source used for subsequent analysis in observational research. As such, they must be created with great diligence. Usually researchers will jot down brief notes during an observation and afterwards elaborate in as much detail as they can recall. Field notes have an important reflective component. In addition to the factual descriptions, researchers include comments about their feelings, reactions, hunches, speculations and working theories or interpretations. The content of field notes, therefore, usually includes: descriptions of the setting, people and activities; direct quotations or paraphrasing of what people said; and the observer's reflections. ${ }^{(23)}$ Field notes are time-consuming when done well - even a single hour of observation can lead to several hours of reflective documentation.

An important aspect to consider when designing observationbased research is the 'observer effect', also known as the Hawthorne effect, more recently reframed as 'participant reactivity' by health professions education researchers Paradis and Sutkin. ${ }^{(24)}$ The Hawthorne effect is conventionally defined as "when observed participants act differently from how they would act if the observer were not present". ${ }^{(25)}$ Researchers have implemented a number of strategies to mitigate this effect, including prolonged embedding of the observer, efforts to 'fit in' through dress or comportment, and careful recording of explicit instances of the effect. (24) However, Paradis and Sutkin found that instances of the Hawthorne effect, as conventionally defined, have never been described in qualitative research manuscripts in the health professions education field, perhaps because, as they speculate, healthcare workers and trainees are accustomed to being observed. Based on this, they argued that researchers should worry less about mitigating the Hawthorne effect and instead invest in interpersonal relationships at their study site to mitigate the effects of altered behaviour and draw on theory to make sense of participants' altered behaviour. ${ }^{(23)}$ Combining

\section{Box 3. Combining interviews and observations:}

It is common to combine these two methods. For instance, we used observations in the operating room to inform in-depth interviews with surgeons about their intraoperative judgements. During surgical procedures of many hours' duration, many challenging and uncertain moments could occur that are of relevance to our study. Surgeons were often unable to describe all of them in detail in response to interview questions. Observing the procedures allows us to create specific, field note-informed interview probes, which supported surgeon participants to richly elaborate on the details of the situation beyond their initial recollection. ${ }^{(29)}$

interviewing and observation is also common in qualitative research (Box 3).

\section{WHAT ARE THE COMMON METHODS OF QUALITATIVE DATA ANALYSIS?}

Qualitative data almost invariably takes the form of text; an interview is turned into a transcript and an observation is rendered into a field note. Analysing these qualitative texts is about uncovering meaning, developing understanding and discovering insights relevant to the research question. Analysis is not separated from data collection in qualitative research, and begins with the first interview, the first observation or the first reading of a document. In fact, the iterative nature of data collection and analysis is a hallmark of qualitative research, because it allows the researcher's emerging insights about the study phenomena to inform subsequent rounds of data collection (Box 4).

Data that has been analysed while being collected is both parsimonious and illuminating. However, this process can extend indefinitely. There will always be another person to interview or another observation to record. Deciding when to stop depends on both practical and theoretical concerns. Practical concerns include deadlines and funding. More importantly, the decision should be guided by the theoretical concern of sufficiency. ${ }^{(26)}$ Sufficiency occurs when new data does not produce new insights into the phenomenon, in other words, when you keep hearing and seeing the same things you have heard and seen before.

Qualitative data analysis is primarily inductive and comparative. The overall process of data analysis begins by identifying segments in the data that are responsive to the

\section{Box 4. The iterative process of analysis:}

You sit down at the dining room table with nothing more than the transcript of your first interview, or the field note of your first observation. You review the purpose of your study. You read and reread the data, making notes in the margins, commenting on the data. You write a separate memo to yourself capturing your reflections, tentative themes, hunches, ideas and things to pursue that are derived from this first set of data. You note things you want to ask, observe or look for in your next round of data collection. After your second interview, you compare the first set of data with the second. This comparison informs the next data collected, and so on. Months later, as you sit down to analyse and write up your findings, you have a set of tentative categories or themes - answers to your research questions from which to continue working. ${ }^{(14)}$ 
research question. The next step is to compare one segment with the next, looking for recurring patterns in the data set. During this step, the focus is on sorting the raw data into categories that progressively build a coherent description or explanation of the phenomenon under study. This process of identifying pieces of data and grouping them into categories is called coding. ${ }^{(14)}$ Once a tentative scheme of categories is derived, it is applied to new data to see whether those categories continue to exist or not, or whether new categories arise - this step determines whether sufficiency has been reached. The final step in the analysis is to think about how categories interrelate. At this point, the analysis moves to interpreting the meaning of these categories and their interrelations. ${ }^{(12)}$

The process for data analysis laid out in this section is a basic inductive and comparative analysis strategy that is suitable for analysing data for most interpretive qualitative research methodologies, including the four featured in this paper phenomenology, grounded theory, ethnography and case study - as well as others such as narrative analysis and action research. While each methodology attends to specific procedures, they all share the use of this basic inductive/comparative strategy. Overall, analysis should be guided by methodology, but different analytical procedures can be creatively combined across methodologies, as long as this combining is explicit and intentional. ${ }^{(27)}$

\section{WHAT ARE SOME CURRENT INNOVATIONS IN QUALITATIVE RESEARCH?}

Understanding the complex factors that influence clinical practice and medical education is not an easy research task. Many important issues may be difficult for the insider to articulate during interviews and impossible for the outsider to 'see' during observation. Innovations to address these challenges include guided walks, ${ }^{(28)}$ photovoice ${ }^{(29)}$ and point-of-view filming. ${ }^{(30)}$ Our own research has drawn intensively on the innovation termed 'rich pictures' to explore the features and implications of complexity in medical education. ${ }^{(31)}$ In one study, we asked medical students to draw pictures of clinical cases that they found complex: an exciting case and a frustrating one. ${ }^{(32)}$ Participants were given 30-60 minutes on their own to reflect on the situation and draw their pictures. This was followed by an in-depth interview using the pictures as triggers to explore the phenomenon under study - in this case, students' experiences of and responses to complexity during their training.

Such innovations hold great promise for qualitative research in medical education. For instance, rich pictures can reveal emotional and organisational dimensions of complex clinical experiences, which are less likely to be emphasised in participants' traditional interview responses. ${ }^{(33)}$ Methodological innovations, however, bring new challenges: they can be timeintensive for participants and researchers; they require new analytical procedures to be developed; and they necessitate efforts to educate audiences about the rigour and credibility of unfamiliar approaches.

\section{WHAT ARE THE PRINCIPLES OF RIGOUR IN QUALITATIVE RESEARCH?}

Like quantitative research, qualitative research has principles of rigour that are used to judge the quality of the work. ${ }^{(34)}$ Here, we discuss principles that appear in most criteria for rigour in the field: reflexivity, adequacy, authenticity, trustworthiness and resonance (Box 5).

The main data collection tool in qualitative research is the researcher. We talk to participants, observe their practices and interpret their documents. Consequently, a critical feature of rigour in qualitative data collection is researcher reflexivity: the ability to consider our own orientations towards the studied phenomenon, acknowledge our assumptions and articulate regularly our impressions of the data. ${ }^{(35)}$ Only this way can we assure others that our subjectivity has been thoughtfully considered and afford them the ability to judge its influence on the work for themselves. Qualitative research does not seek to remove this subjectivity; it treats research perspective as unavoidable and enriching, not as a form of bias to purge.

Every qualitative dataset is an approximation of a complex phenomenon - no study can capture all dimensions and nuances of situated social experiences, such as medical students' negotiations of professional dilemmas in the clinical workplace. Therefore, two other important criteria of rigour relate to the adequacy and authenticity of the sampled experiences. Did the research focus on the appropriate participants and/or situations? Was the size and scope of the sample adequate to represent the scope of the phenomenon? ${ }^{(36)}$ Was the data collected an authentic reflection of the phenomenon in question? Qualitative researchers should thoughtfully combine different perspectives, methods and data sources (a process called 'triangulation') to intensify the richness of their representation. ${ }^{(37)}$ We should endeavour to draw on data in our written reports such that we provide what sociologist Geertz has termed a sufficiently 'thick' description ${ }^{(38)}$ for readers to judge the authenticity of our portrayal of the studied phenomenon.

Qualitative analysis embraces subjectivity: what the researcher 'sees' in the data is a product both of what participants told or showed us and of what we were oriented to make of those stories and situations. To some degree, a rhetorician will always see rhetoric and a systems engineer will always see systems. To fulfil the rigour criteria of trustworthiness, qualitative

\section{Box 5. Principles of rigour in qualitative research}

- Reflexivity: was the researcher's orientation and its influence on the work considered?

- Adequacy: is the data sufficient to allow robust insight into the studied phenomenon?

- Authenticity: does the data provide an authentic depiction of the social phenomenon being studied?

- Trustworthiness: are the analytical procedures systematic and clearly described?

- Resonance: are the findings and interpretations meaningful and useful to those who live this social experience being studied? 
analysis should also be systematic and held to a principle of trustworthiness, which dictates that we should clearly describe: (a) what was done by whom during the inductive, comparative analytical process; (b) how the perspectives of multiple coders were negotiated; (c) how and when theoretical lenses were brought to bear in the iterative process of data collection and analysis; and (d) how discrepant instances in the data - those that fell outside the dominant thematic patterns - were handled.

Finally, the ultimate measure of quality in qualitative research is the resonance of the final product to those who live the social experience under study. ${ }^{(4)}$ As qualitative researchers presenting our work at conferences, we know we have met this bar if our audiences laugh, nod or scowl at the right moments, and if their response at the end is "You nailed it. That's my world. But you've given me a new way to look at it". The situatedness of qualitative research means that its transferability to other contexts is always a matter of the listener/reader's judgement, based on their consideration of the similarities and differences between the research context and their own. Thus, there is a necessity for qualitative research to sufficiently describe its context, so that consumers of the work have the necessary information to gauge transferability. Ultimately, though, transferability remains an open question, requiring further inquiry to explore the explanatory power of one study's insights in a new setting.

\section{WHAT ELSE IS THERE TO KNOW?}

This overview of qualitative research in medical education is not exhaustive. We have been purposefully selective, discussing in depth some common methodologies and methods, and leaving aside others. We have also passed over important issues such as qualitative research ethics, sampling and writing. There is much, much more for readers to know! Our selectivity notwithstanding, we hope that this paper will provide an accessible introduction to some qualitative essentials for readers who are new to this research domain, and that it may serve as a useful resource for more experienced readers, particularly those who are doing a qualitative research project and would like a better sense of where their work sits within the broader field of qualitative approaches.

\section{REFERENCES}

1. Kuper A, Reeves S, Levinson W. An introduction to reading and appraising qualitative research. BMJ 2008; 337:a288.

2. Denzin NK, Lincoln YS. Introduction: The discipline and practice of qualitative research. In Denzin NK, Lincoln YS, eds. The Sage Handbook of Qualitative Research. 3rd ed. Thousand Oaks, CA: Sage Publications, 2005: 1-33.

3. Carter SM, Little M. Justifying knowledge, justifying method, taking action: epistemologies, methodologies, and methods in qualitative research. Qual Health Res 2007; 17:1316-28.

4. Charmaz K. Constructing Grounded Theory. 2nd ed. Thousand Oaks, CA: Sage Publications, 2014.

5. Apramian T, Cristancho S, Watling C, Lingard L. (Re)Grounding grounded theory: a close reading of theory in four schools. Qual Res 2017; 17:359-76.

6. Schutz A. Subjective and Objective Meaning. In: The phenomenology of the social world. New York: Northwestern University Press, 1967.

7. Reeves S, Albert M, Kuper A, Hodges BD. Why use theories in qualitative research? BMJ 2008; 337:a949.
8. Laverty SM. Hermeneutic phenomenology and phenomenology: a comparison of historical and methodological considerations. Int J Qual Methods 2003; 2:21-35.

9. Atkinson P, Pugsley L. Making sense of ethnography and medical education. Med Educ 2005; 39:228-34.

10. Reeves S, Kuper A, Hodges BD. Qualitative research methodologies: ethnography. BMJ 2008; 337:a1020.

11. Baxter P, Jack S. Qualitative case study methodology: study design and implementation for novice researchers. Qual Rep 2008; 13:544-59.

12. Watling CJ, Lingard L. Grounded theory in medical education research: AMEE Guide No. 70. Med Teach 2012; 34:850-61.

13. Hopkins RM, Regehr G, Pratt DD. A framework for negotiating positionality in phenomenological research. Med Teach 2017; 39:20-5.

14. Taylor TS, Nisker J, Lingard L. To stay or not to stay? A grounded theory study of residents' postcall behaviors and their rationalizations for those behaviors. Acad Med 2013; 88:1529-33.

15. McLachlan E, King N, Wenger E, Dornan T. Phenomenological analysis of patient experiences of medical student teaching encounters. Med Educ 2012; 46:963-73.

16. Reeves S, McMillan SE, Kachan N, et al. Interprofessional collaboration and family member involvement in intensive care units: emerging themes from a multi-sited ethnography. J Interprof Care 2015; 29:230-7.

17. Whitehead C, Selleger V, van de Kreeke J, Hodges B. The 'missing person' in roles-based competency models: a historical, cross-national, contrastive case study. Med Educ 2014; 48:785-95.

18. Merriam SB, Tisdell EJ. Qualitative Research: A Guide to Design and Implementation. 4th ed. Jossey-Bass, 2015.

19. Rubin HJ, Rubin IS. The Responsive Interview as an Extended Conversation. In: Qualitative Interviewing: The Art of Hearing Data. Sage Publications, 2011.

20. Bernabeo EC, Holmboe ES, Ross K, Chesluk B, Ginsburg S. The utility of vignettes to stimulate reflection on professionalism: theory and practice. Adv Health Sci Educ Theory Pract 2013; 18:463-84.

21. Goldszmidt M, Faden L, Dornan T, et al. Attending physician variability: a model of four supervisory styles. Acad Med 2015; 90:1541-6.

22. Heath C, Luff $P$, Svensson MS. Video and qualitative research: analysing medical practice and interaction. Med Educ 2007; 41:109-16.

23. Emerson RM, Fretz RI, Shaw LL. Writing Ethnographic Fieldnotes. University of Chicago Press, 2011.

24. Paradis E, Sutkin G. Beyond a good story: from Hawthorne Effect to reactivity in health professions education research. Med Educ 2017; 51:31-9.

25. Ng S, Lingard L, Kennedy TJ. Qualitative Research in Medical Education: Methodologies and Methods. Understanding Medical Education: Evidence, Theory and Practice. Oxford, UK: Wiley-Blackwell, 2013: 371-84.

26. Morse JM. Strategies for sampling. In: Morse JM, eds. Qualitative Nursing Research: A Contemporary Dialogue. Newbury Park, CA: Sage Publications, 1991: 127-45.

27. Varpio L, Martimianakis MA, Mylopoulos M. Qualitative research methodologies: embracing methodological borrowing, shifting and importing. In: Cleland J, Durning SJ, eds. Researching Medical Education. Oxford, UK: Wiley-Blackwell, 2015: 245-59.

28. Dubé TV, Schinke RJ, Strasser R, Lightfoot N. Interviewing in situ: employing the guided walk as a dynamic form of qualitative inquiry. Med Educ 2014; 48:1092-100.

29. LaDonna KA, Venance SL. Picturing the experience of living with myotonic dystrophy (DM1): a qualitative exploration using photovoice. J Neurosci Nurs 2015; 47:285-95.

30. Skinner J, Gormley GJ. Point of view filming and the elicitation interview. Perspect Med Educ 2016; 5:235-9.

31. Cristancho SM, Bidinosti SJ, Lingard L, et al. What's behind the scenes? Exploring the unspoken dimensions of complex and challenging surgical situations. Acad Med 2014; 89:1540-7.

32. Helmich E, Diachun L, Joseph R, et al. 'Oh my God, I can't handle this!': trainees' emotional responses to complex situations. Med Educ 2018; 52:206-15.

33. Cristancho S, Bidinosti S, Lingard L, et al. Seeing in different ways introducing "rich pictures" in the study of expert judgment. Qual Health Res 2015; 25:713-25.

34. Tracy SJ. Qualitative quality: eight "big-tent" criteria for excellent qualitative research. Qual Inq 2010; 16:837-51.

35. Guillemin M, Gillam L. Ethics, reflexivity, and "ethically important moments" in research. Qual Inquiry 2004; 10:261-80.

36. Morse JM. Determining sample size. Qual Health Res 2000; 10:3-5.

37. Thurmond VA. The point of triangulation. J Nurs Scholarsh 2001; 33:253-8.

38. Geertz C. Thick Description: Toward an Interpretive Theory of Culture. In: The Interpretation of Cultures: Selected Essays. New York: Basic Books, 1973. 